\title{
Life threatening arrhythmias: Knowledge and skills among nurses working in critical care settings at Muhimbili National Hospital, Dar es Salaam, Tanzania
}

\author{
DINNAH I. RUHWANYA ${ }^{*}$, EDITH A.M. TARIMO² and MENTI NDILE ${ }^{2}$ \\ ${ }^{1}$ Emergency Medicine Department, Muhimbili National Hospital, P O Box 65000, Dar es Salaam, Tanzania \\ ${ }^{2}$ Muhimbili University of Health and Allied Sciences, P.O Box 65004, Dar es Salaam, Tanzania
}

\begin{abstract}
Introduction: A life threatening arrhythmia is a medical condition that requires immediate intervention, or it can cost a patient's life. However, there is limited understanding of nurses' knowledge and skills in identification and care provided to patients with life threatening arrhythmias in Tanzania. The objective of this study was to assess knowledge and skills of nurses in identifying life threatening arrhythmias and the required patient care.

Methods: A descriptive cross-sectional study was conducted to assess the knowledge and skill level of nurses in identification of life threatening arrhythmias and the required patient care, as well as exploring the barriers in acquiring and implementing particular knowledge and skills. A convenient sample of 141 nurses working in critical care areas at Muhimbili National Hospital, were recruited.

Results: The majority (44\%) of the participants were 31-40 years old and more than three quarters were females. The majority (60\%) scored highly when tested about their knowledge of life threatening arrhythmia identification. However, the majority of nurses (84.4\%) scored poorly when being observed on their skills. A total of $116(82.3 \%)$ nurses were able to identify asystole on an electrocardiogram strip, and demonstrated a high level of knowledge (95\%) in the nursing care of patients in asystole. Although they demonstrated poor skills in general, nurses were competent (97.9\%) in electrode placement on the patient's chest before connecting the patient to the cardiac monitor. The overwhelming workload was identified by many (68.8\%) as the major barrier in acquiring and implementing knowledge and skills.

Conclusion: Although the majority of the participants scored highly in their level of knowledge regarding life threatening arrhythmias, they scored poorly in most of the observed skills when identifying and treating this patient group. It is important that hospital administration take into consideration the identified areas of deficiency and work to improve the skills among nurses and enhance optimal care of patients.
\end{abstract}

Keywords: arrhythmias, knowledge, skills, nurses, critical care settings, Tanzania

\section{Introduction}

Arrhythmias are abnormal heart rhythms due to disturbances in heart automaticity and/or abnormal heart conduction which cause a reduction in cardiac output, a change in heart rate thus affecting tissue perfusion (Diehl, 2011). Any impulse originating outside the sino-atrial node can cause an abnormal heart rhythm. Much of the literature considers ventricular tachycardia, ventricular fibrillation, pulseless electrical activity, complete heart block, and asystole to be the most common types of life threatening arrhythmias (Leijdekkers \& Gay, 2006; Diehl, 2011). Cardio-vascular conditions are becoming common ailments in Tanzania. In 2012, 18 to 24\% of deaths were in Tanzania were due to non-communicable diseases and $9 \%$ of those were due to a cardiovascular conditions, with predisposing factors being obesity, tobacco use, alcohol consumption, and hypertension (Mayige et al., 2012). In Tanzania, known as developing country, there was no dedicated cardiac care centre until 2005, when the government sent some staff outside the country for formal cardiac training. Among

\footnotetext{
*Correspondence E-mail: rdinnah@gmail.com
} 
those staff sent to different countries to gain more advanced cardiac knowledge, were nurses of different cadres (Nyawawa et al., 2005).

Currently, an electrocardiogram (ECG) is normally used as the first tool for initial screening of cardiovascular disorders and non-invasive diagnosis of life threatening arrhythmias in clinical practice (Sayadi \& Shamsollahi, 2011). In critical care settings ECG's provide nurses with information about the patient's electro-cardiac record in a manner that is easy and fast to use. Nurses who care for critically ill patients need to have a thorough understanding of the information provided by the ECG, which includes heart rate, regularity of the rhythm, interval measurements and characteristics of each individual wave form of the heart.

In critical care settings, bedside nurses play a critical role in arrhythmia identification and management. The current practice is that nurses gather data, and notify the physician, who makes treatment decisions based on the rhythm interpretation of the nurse, or institutes pharmacologic and counter shock therapies consistent with unit-specific protocols or algorithms (Keller \& Raines, 2005). Thus, efforts are needed to increase and maintain the knowledge of ECG interpretation since it has been observed that a nurse's knowledge and skills in ECG interpretation diminish with time (Nolan et al.,2010). Although there is no one specific method for teaching critical care nurses about arrhythmias, several approaches have been used to assist nurses to increase their knowledge and skills (Lak et al., 2013). In a combined interactive web-based collaborative learning activity and validation of staff's competency using a skills checklist, Schultz (2010) suggested that hospital administrators should encourage nurses to participate in a clinical practice development programme, to increase their knowledge and skills in arrhythmia monitoring. This can encourage nurses to enjoy learning, which makes it easier for them to master the skill.

Currently, there is limited information regarding nurse's knowledge and skills in identification of life threatening arrhythmias and providing care for this group of patients in Tanzania. The objective of this study was to assess knowledge and skills of nurses in identifying life threatening arrhythmias and the required patient care at the Muhimbili National Hospital (MNH) in Tanzania.

\section{Materials and Methods}

\section{Study areas and study population}

The study was conducted at Muhimbili National Hospital in Dar es Salaam, Tanzania. MNH is the only national referral hospital and a university teaching hospital. The study involved the Emergency Medicine Department (EMD), Coronary Care Unit (CCU), Main Intensive Care Unit (ICU), Highly Dependent Unit (Ward -1 HDU), and Cardiothoracic Intensive Care Unit (CICU). A total of 141 nurses were involved, representing $83 \%$ of all nurses working in critical care settings at $\mathrm{MNH}$.

\section{Study design and sampling procedure}

This was a descriptive cross-sectional study using quantitative approach and involved nurses. A selfadministered questionnaire was used to collect information regarding knowledge, while an observational checklist was used to record the skills. The target population was finite to include 170 nurses. The study increased representativeness by including any nurse working in a critical care setting during data collection. A convenient sampling strategy was employed, whereby any nurse who was on duty and agreed to participate was included.

\section{Data collection}

A pre-tested self-administered questionnaire was used to collect information regarding knowledge, while an observational checklist was used to record the skills. The questionnaire comprised of socio- 
demographic data, then questions testing knowledge about life threatening arrhythmias through interpreting ECG strips and the proper management of these patients. The questionnaire also investigated the barriers hindering nurses from acquiring a high level of knowledge and skill when caring for patients with life-threatening arrhythmias. The observational checklist was used to collect data on the skills related to connection of the patient to the cardiac monitor and ECG machine through proper lead placement, skills in cardiopulmonary resuscitation (CPR) and the procedure of defibrillation including connection, pad placement and delivering shocks. Data collection took place during April 2016.

\section{Data analysis}

Data was analysed by using the Statistical Package for Social Sciences (SPSS) Version 20.0 program. With regard to the level of knowledge, correct answers for the each of the eight questions were totalled then converted into a percentage. All 9 items on the observational checklist regarding skills were weighted with the digits 0-2. Weighting of 2 indicated the nurse was "competent", 1 indicated "needs improvement" and o indicated "not competent". The method used for skills evaluation was simulation. The stated weight was also converted into a percentage which ranged from $0-100 \%$. The levels of knowledge and skills were classified in two ways: $100 \%-50 \%=$ high and $49 \%-0 \%=$ poor. ChiSquare and Fisher's exact tests were performed to assess the association between socio-demographic factors and the level of knowledge and skills. The probability of $p \leq 0.05$ was considered significant.

\section{Ethical considerations}

Ethical clearance was obtained from Muhimbili University of Health and Allied Sciences Institutional Review Board (Ref. No MU/PGS/SAEC/Vol.X1V). The permission to conduct the study at the hospital was obtained from Muhimbili National Hospital Management (Ref. No MNH/TRC/2016/672). All potential participants received information about the study while on duty, and they were provided with information on the potential risks and benefits of participating. They were asked to sign a written informed consent form prior to the study and this was voluntary. To ensure confidentiality, a unique code was used on the data collection tools instead of individual names.

\section{Results}

\section{Socio-demographic characteristics}

A total of 141 nurses participated in the study. Majority of participants (44.0\%) were aged 31-40 years, with a mean age of $34.1 \pm 7.3$ years. More than three quarters of participants were females (79.4\%). Majority (70. $2 \%$ ) had a diploma in nursing as their highest level of education. Majority (56.7\%) had worked for five or more years in their current working situation (Table 1). 
Table 1: Socio-demographic characteristics

\begin{tabular}{llll}
\hline Variable & Response & Frequency (N=141) & Percentage \\
\hline Age (years) & $20-30$ & 57 & $40.4 \%$ \\
& $31-40$ & 62 & $44.0 \%$ \\
& $41-50$ & 17 & $12.1 \%$ \\
Sex & $51-60$ & 5 & $3.5 \%$ \\
Qualification in Nursing & Male & 29 & $20.6 \%$ \\
& Female & 112 & $79.4 \%$ \\
& Certificate & 14 & $9.9 \%$ \\
Working area & Diploma & 99 & $70.2 \%$ \\
& Bachelor & 20 & $14.2 \%$ \\
& Masters & 8 & $5.7 \%$ \\
& Main ICU & 23 & $16.3 \%$ \\
& EMD & 77 & $54.6 \%$ \\
Working experience in the current area (years) & CCU & 7 & $5 \%$ \\
& HDU-Ward 1 & 21 & $14.9 \%$ \\
& Cardiothoracic ICU & 13 & $9.2 \%$ \\
Type of training attended & $3-4$ & 41 & $29.1 \%$ \\
& S and above & 80 & $14.2 \%$ \\
Equipment available & ECG & 90 & $56.7 \%$ \\
& BLS & 118 & $63.8 \%$ \\
& ACLS & 24 & $83.7 \%$ \\
& Cardiac monitor & 141 & $17.0 \%$ \\
& 12 lead EKG & 77 & $100 \%$ \\
& Others & 10 & $54.6 \%$ \\
& & $7.1 \%$ \\
\hline
\end{tabular}

Key: ICU= intensive care unit; EMD=Emergency Medicine Department; CCU=Coronary Care Unit; HDU=High Dependency Unit; BLS=Basic Life Support; ACLS=Advanced Cardiac Life Support; EKG=Electrocardiography

\section{Knowledge of nurses}

The level of knowledge regarding life threatening arrhythmias among study participants was generally high. Of the 141 nurses, 85 (60\%) scored a high level of overall knowledge, answering more than half of the questions correctly. The mean score was 56.0\% (SD: 17.4\%). Of 141 nurses, 56 (40\%) scored a low level of knowledge as their percentage of correct answers was less than $49.9 \%$. The minimum score was $11.1 \%$ and the maximum score was $88.8 \%$, while the majority scored between $44.4 \%$ and $55.5 \%$. Detailed results show that majority of participants (82.3\%) scored a high level of knowledge on identification of asystole on the ECG strip. Almost all (95\%) demonstrated a high level of knowledge on the nursing care of patients with this arrhythmia. While on the other hand, a low knowledge score was noted regarding complete heart block in the ECG strip, with only $38.3 \%$ of participants being able to identify it correctly (Table 2). 
Table 2: Percentage distribution of nurses who got correct answers, using the individual knowledge questions $(\mathrm{N}=141)$

\begin{tabular}{lll} 
Question type & Frequency & Percentage \\
\hline Correct ECG strip identification (ventricular tachycardia) & 66 & $44.4 \%$ \\
Correct ECG strip identification (asystole) & 116 & $82.3 \%$ \\
Correct ECG strip identification (complete heart block) & 54 & $38.3 \%$ \\
Correct ECG strip identification (ventricular fibrillation) & 55 & $39 \%$ \\
Appropriate nursing action if pulseless electrical activity (PEA) and asystole were & 134 & $95 \%$ \\
identified on the cardiac monitor & & \\
Appropriate measures indicated for unstable ventricular tachycardia with a pulse & 56 & $39.7 \%$ \\
Appropriate nursing action for a patient with pulseless ventricular tachycardia & 93 & $66 \%$ \\
(VT)/ ventricular fibrillation(VF) & & \\
The first action taken by the nurse after identifying new ventricular tachycardia & 83 & $58.9 \%$ \\
\hline
\end{tabular}

\section{Level of nursing skill}

The level of skill of nurses regarding life threatening arrhythmias was generally poor. Of the 141 nurses, $119(84.4 \%)$ scored a low skill level, which ranged from o to $49.9 \%$. This means only $15.6 \%$ scored highly. The minimum score was $11.5 \%$ and the maximum score was $92.3 \%$. Although generally the participants had a poor skills score overall, the majority (97.9\%) demonstrated competent skills in electrode placement on the patient's chest before connecting the patient to the cardiac monitor. Over three quarters (78.7\%) of the nurses were competent in CPR skills. However, a low level of skill was observed in skin preparation before application of electrodes, as $94.3 \%$ were not competent (Table 3 ).

Table 3: Competence level of nurses by individual skill observed.

\begin{tabular}{|c|c|c|c|}
\hline \multirow[t]{2}{*}{ Skill type } & \multicolumn{3}{|c|}{ Frequency and Percentage } \\
\hline & Competent & Needs improvement & Not competent \\
\hline Skin preparation before application of electrodes & 0 & $8(5.7 \%)$ & $133(94 \cdot 3 \%)$ \\
\hline Lead placement for cardiac monitor & $138(97 \%)$ & $3(2.1)$ & 0 \\
\hline Lead placement for 12 -lead ECG & $74(52.5 \%)$ & $2(1.1 \%)$ & $65(46.1 \%)$ \\
\hline Conduction of ECG & $80(56.7 \%)$ & $1(0.7 \%)$ & $60(42.6 \%)$ \\
\hline Identification of P wave on ECG & $13(9.2 \%)$ & $28(19.9 \%)$ & $100(70.9 \%)$ \\
\hline Identification of QRS complex & $16(11.3 \%)$ & $48(34 \%)$ & $77(54.6 \%)$ \\
\hline Conduction of CPR procedure & $111(78.7 \%)$ & $27(19.1 \%)$ & $3(2.1 \%)$ \\
\hline Connecting and charging the defibrillator machine & $8(5.7 \%)$ & $52(36.9 \%)$ & $81(57.4 \%)$ \\
\hline Pad/paddle placement during defibrillation & $12(8.5 \%)$ & $53(37.6 \%)$ & $76(53.9 \%)$ \\
\hline
\end{tabular}

Association between socio-demographic characteristics and the level of knowledge and skills An association was found between the nurses' level of knowledge and educational qualifications, as well as between having a high level of knowledge and having attending Advanced Cardiac Life Support $(A C L S)$ training $(p<0.05)$. A significant association was found between skills observed and educational qualifications, along with skills observed and ECG training $(P<0.001)$ (Table 4$)$. 
Table 4: Comparison between knowledge and skills scores and independent variables

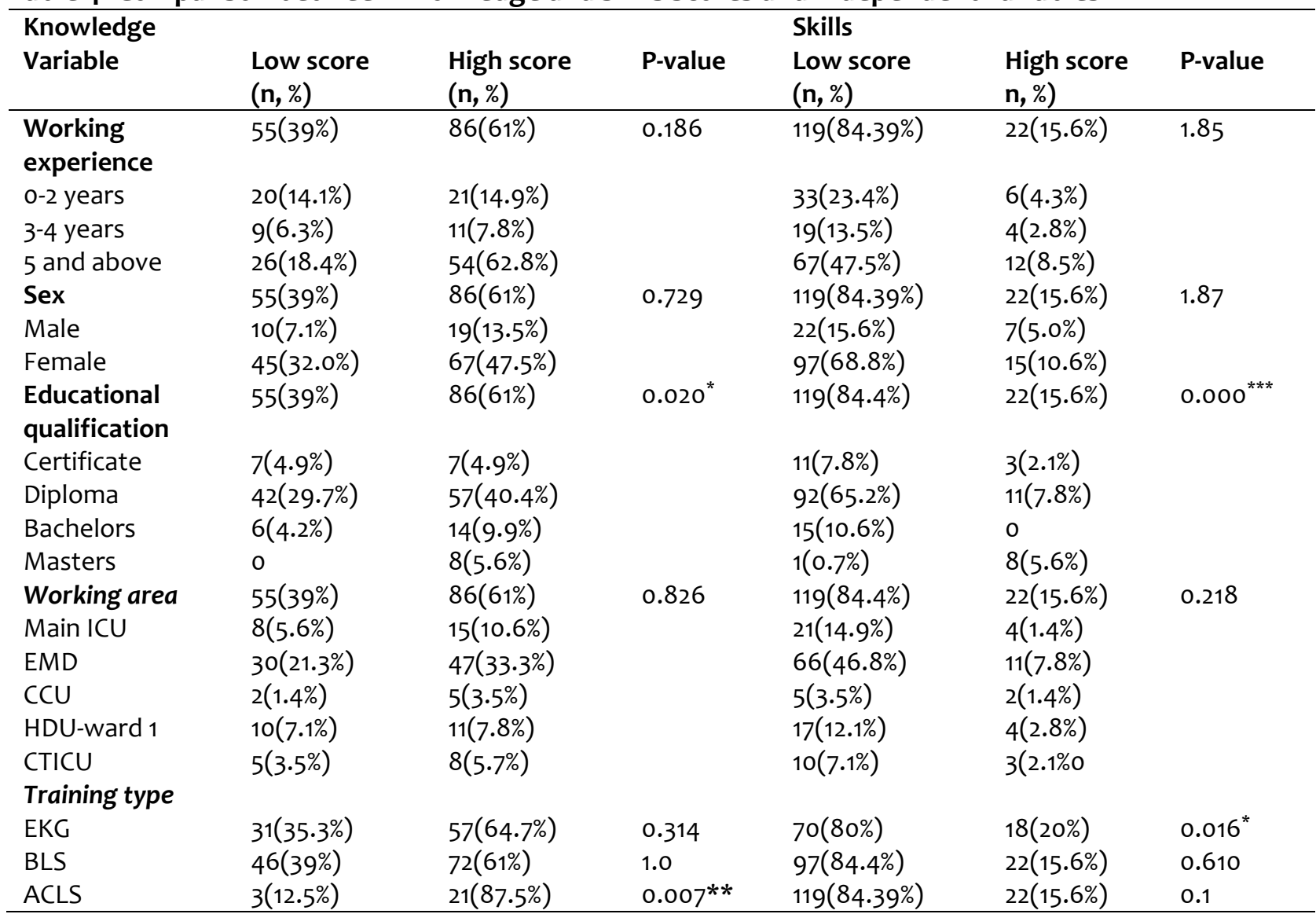

Significance marked as ${ }^{*} \mathrm{P}<0.05, \mathrm{P}<* * 0.01, * * * \mathrm{P}<0.001 ;$ Key: EKG=Electrocardiography; BLS=Basic Life Support;

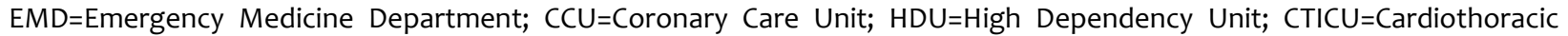
Intensive Care Unit; ACLS=Advanced Cardiac Life Support

Table 5: Barriers encountered by nurses when acquiring knowledge and skills

\begin{tabular}{|c|c|c|}
\hline Variable & Frequency & $\%$ \\
\hline $\begin{array}{l}\text { No polices and/or standards available for nurses, explaining how to care for patients } \\
\text { with life threatening arrhythmias }\end{array}$ & 60 & $30.2 \%$ \\
\hline Lack of team work when caring for patients with life threatening arrhythmias & 54 & $38.3 \%$ \\
\hline Stress caused by overwhelming workload & 97 & $68.8 \%$ \\
\hline Lack of confidence /feeling inadequate in the area of knowledge and skills & 48 & $34 \%$ \\
\hline Lack of adequate space to practice skills & 8 & $5.7 \%$ \\
\hline Other reasons - lack of not practicing frequently & 4 & $2.8 \%$ \\
\hline $\begin{array}{l}\text { Lack of mandatory training about how to care for patients with life threatening } \\
\text { arrhythmias }\end{array}$ & 72 & $51.1 \%$ \\
\hline $\begin{array}{l}\text { No guidelines/algorithms available about the specific treatment required when caring } \\
\text { for patients with life threatening arrhythmias }\end{array}$ & 62 & $44 \%$ \\
\hline $\begin{array}{l}\text { Inadequate monitors or machines to detect life threatening arrhythmias, compared to } \\
\text { the number of patients }\end{array}$ & 85 & $60.3 \%$ \\
\hline Lack of incentive to teach others, of those who are competent & 57 & $40.4 \%$ \\
\hline Other reasons - lack of equipment for practicing including a manikin & 1 & $0.7 \%$ \\
\hline
\end{tabular}




\section{Barriers in acquiring knowledge and skills to care for patients}

Of 141 participants, 121 (86.5\%) reported they had encountered barriers when acquiring knowledge and skills regarding life threatening arrhythmias. The major barriers found were stress caused by overwhelming workload, identified by $68.8 \%$ of participants; followed by inadequate monitors or machines available to detect life threatening arrhythmias identified by $60.3 \%$ (Table 5 ).

\section{Discussion}

Majority of the participants had a diploma in nursing, and most of them had worked for five years or more in the critical care setting. They had training in Basic Life Support (BLS) and ECG interpretation. However, only a few had attended an advanced cardiac life support training course. Overall, the majority scored a high level of knowledge when caring for patients with life threatening arrhythmias. However, only a few demonstrated practical application skills in the same area. The available resources, such as training and equipment enhanced knowledge amongst participants. The more educational qualifications a participant had, the higher his/her score in both knowledge and skills.

This study has shown that the nurses have a high level of knowledge when interpreting life threatening arrhythmias. This may be due to the fact that most of the nurses had been educated from diploma level and above, thus having been taught the theory in the classroom. Also, availability of equipment to assess for life threatening arrhythmias, like cardiac monitors and an ECG machine within the work settings may have increased the nurses' level of knowledge, as they are able to interpret arrhythmias regularly. Similar findings have been reported from India (Mohan, 2010). The fact that the majority of the participants scored highly in the area of knowledge regarding identification of asystole on the ECG strip implies that the available resources in terms of equipment and training were effectively utilized for this particular rhythm. Also, in a similar study, Mohan (2010) showed that 100\% of the participants interpreted ECG strips of asystole correctly. Our current study has found that nurses have a low level of knowledge when interpreting complete heart block and about how to care for patients with this arrhythmia. Similar results have been reported from a study in the USA (Keller \& Raines 2005).

Overall the current results demonstrate a tremendous improvement in the number of nurses who have attended BLS and ACLS trainings. Hilary's study (2012) identified only 31 of 73 (42\%) participants had received BLS training and $12(16 \%)$ had received ACLS training within similar settings at $\mathrm{MNH}$, excluding paediatric ICU. According the Gabbot et al. (2005) it is up to the health care institutions to ensure their staff members receive adequate training on a regular basis to maintain their levels of competence. The finding of this study also indicates a statistical association between ACLS training and the level of knowledge. This might be influenced by the advanced life saving procedures which are included in the course. Similarly, Passali et al. (2011) found that respondents who reported having ACLS training, scored significantly better in all ACLS questions compared to those with no previous ACLS training. The participants demonstrated a low level of skill when identifying life threatening arrhythmias. To provide appropriate and safe care, nurses need to have an adequate to excellent level of skill, in order to minimize a patient's suffering and complications (Keller \& Raines 2005).

Incompetence in preparation of patients before application of the electrodes onto the skin is witnessed in the present study. Poor skin preparation increases skin impedance and minimizes conduction of the cardiac signal available to capture an impulse. Rubbing of the skin and shaving are both recommended by the manufacturer of the electrodes, to ensure optimal electrode attachment and appropriate signals. In Norway, Pettersen et al. (2014), reported that, among patients who required shaving, only less than half were shaved; while of those who needed their skin wiped to 
remove oil, debris or any dirt, only a few were rubbed with a cloth. Failure to complete skin preparation before electrode placement may cause inappropriate monitoring alarms for patients with continuous ECG monitoring.

Despite the availability of a defibrillator in each setting, this study identified a deficit in knowledge and skills when using the machine, including the connection, charging and pad placement. Hebert (2017) highlighted that, nurses need continuous training, which should be done in the working places, especially when learning how to use sophisticated machines. Skills in advanced procedures like defibrillation, 12-lead ECG interpretation and others require considerable repetition in training and clinical practice in order for the provider to be competent. This finding indicates a large gap between nurses' knowledge and skills i.e., though the nurses had a good theoretical knowledge, they lacked the necessary skills to apply the knowledge appropriately. The significant association found between the level of education, and skills and knowledge in caring for patients with life threatening arrhythmias is worth noting. The reason might be that, advances in nursing education increases learning, and the understanding of advanced life saving procedures.

The major barrier found in this study was stress caused by an overwhelming workload. In critical care settings, as reported by Tevington (2011) that the recommended standard of care stated that nurse- patient ratio is one to two(one nurse should care two patients at a time) However, the reality in a critical care settings at $\mathrm{MNH}$ is many times one nurse can care for five to fifteen patients per shift. Lambert \& Lambert (2008) pointed out that excessive workloads are among the leading factors causing substandard care. Another barrier identified was inadequate equipment, such as cardiac monitors or machines. Unavailability or non-functional equipment directly affects the nurse's autonomy, the nature of the workload and the quality of patient care that can be delivered (Bucknall, 2003). The findings of this study are likely to have some limitations. This study was conducted in one referral hospital, and hence the findings might not represent all other referral hospitals in the country. In future, multiple referral hospitals should be involved in order to achieve external validity.

In conclusion, the current study has shown that although nurses working in critical care settings have a high level of knowledge, they have poor skills regarding life threatening arrhythmias. This study calls the hospital management to have a clinical nurse trainer to support and encourage nurses to translate acquired knowledge into practical skills. This can be supported by designing and implementing a continuous professional practical skills programme on life threatening arrhythmia care.

\section{Competing interests}

Authors declare no competing interests.

\section{Authors' contributions}

DIR conceptualized and designed the study, supervised its implementation, performed data analysis and drafted the manuscript. EAMT provided inputs to the study design, implementation and data analysis. MN provided inputs to the study design and reviewed the manuscript. All authors approved the manuscript. 


\section{Acknowledgements}

We acknowledge the study participants, research assistants and MNH for allowing data collection within the hospital premises. The study was funded by Ministry of Health, Community, Development, Gender, Elderly and Children.

\section{References}

Bucknall, T. (2003) The clinical landscape of critical care: nurses' decision-making. Journal of Advanced Nursing 43: 310-319.

Diehl, T.S. (2011) ECG Interpretation Made Incredibly Easy. Philadelphia, Chris Burghardt.

Gabbott, D., Smith, G., Mitchell, S., Colquhoun, M., Nolan, J., Soar, J., Pitcher, D., Perkins, G., Phillips, B., King, B., \& Spearpoint, K. (2005). Cardiopulmonary resuscitation standards for clinical practice and training in the UK. Resuscitation 64: 13-19.

Hebert, R. L. (2017). Initiation of In-hospital CPR: An examination of nursing behaviour within their scope of practice (Doctoral dissertation, Université d'Ottawa/University of Ottawa).

Hillary, A. (2012). Assessment of knowledge and skills of cardiopulmonary resuscitation among nurses working in critical care units at Muhimbili National Hospital, Dar es salaam, Tanzania. http:www.pdfiller.com/jsfiller-desk4/projectld=171785331\&exld=3213\&expBranch=1\#593C72 af73e6b45cf982687ea4d. (Accessed 3 May 2016)

Keller, K.B. \& Raines, D.A. (2005) Arrhythmia knowledge: a qualitative study. Heart Lung 34: 309-316.

Krejcie, R. \& Morgan, D. (1970) Determining sample size for research activities. Educational and Psychological Measurements 30: 607-610.

Lak, K., Zareie, F., Habibzadeh, H., Mohammad, Y., Rahnemoon, K., Zare, H., \& Zaviyehi, M. (2013) A survey on the effect of educational software method of arrhythmias stimulator on the level of knowledge of electrocardiograms interpretation in nurses. Iranian Journal of Critical Care Nursing 6: 173-180.

Lambert, V.A. \& Lambert, C.E. (2008) Nurses' workplace stressors and coping strategies. Indian Journal of Palliative Care 14:38-44.

Leijdekkers, P. \& Gay, V. (2006) Personal Heart Monitoring and Rehabilitation System using Smart Phones. Available at: https://www.computer.org/csdl/proceedings/icmb/2006/2595/00/25950029.pdf ICMP, IEEE.

Mayor, M. (2010) Longman Active Study Dictionary. 2010; Pearson Education India.

Mayige, M., Kagaruki, G., Ramaiya, K. \& Swai, A. (2011) Non communicable diseases in Tanzania: a call for urgent action. Tanzania Journal of Health Research 13 (Suppl 1): 378-386.

Mohan, S. (2010) A study to assess the knowledge regarding interpretation of life threatening arrhythmias and its emergency management among cardiac nurses in SCTIMST, Trivandrum 695011.

Nolan, J. P., Soar, J., Zideman, D. A., Biarent, D., Bossaert, L. L., Deakin, C. \& Böttiger, B. (2010). European resuscitation council guidelines for resuscitation 2010 section 1. Executive summary. Resuscitation 81: 1219-1276.

Nyawawa, E.T.M., Ussiri, E.V., Chillo, P., Waane, T., Lugazia, E., Mpoki, U., Luchemba, R., Wandwi, B., Nyangasa, B., Bgoya, J. \& Mahalu, W. (2010) Cardiac Surgery: One year experience of cardiac surgery at Muhimbili National Hospital, Dar es Salaam, Tanzania. East and Central African Journal of Surgery 15: 111-118. 
Passali, C., Pantazopoulos, I., Dontas, I., Patsaki, A., Barouxis, D., Troupis, G. \& Xanthos, T. (2011) Evaluation of nurses' and doctors' knowledge of basic \& advanced life support resuscitation guidelines. Nurse Education in Practice 11: 365-369.

Pettersen, T.R., Fålun, N. \& Norekvål, T.M. (2014) Improvement of in-hospital telemetry monitoring in coronary care units: an intervention study for achieving optimal electrode placement and attachment, hygiene and delivery of critical information to patients. European Journal of Cardiovascular Nursing 13: 515-523.

Sayadi, O. \& Shamsollahi, M.B. (2011) Life-threatening arrhythmia verification in ICU patients using the joint cardiovascular dynamical model and a Bayesian filter. IEEE Transactions of Biomedical Engineering 58: 2748-2757.

Schultz, S.J. (2010) Dysrhythmia Monitoring Practices of Nurses on a Teletry Unit. Teletry Unit. Florida, Florida Brooks College of Health.

Tevington, P. (2011) Mandatory nurse-patient ratios. Medsurg Nursing 20: 265-268. 